\title{
The Satellites Orientation Method using a Torque Magnetic Drive
}

\author{
B. Samotokin, D. Shostachuk, I. KorobilchuK ${ }^{a, *}$, M. Kachniarz $^{b}$, M. Nowicki $^{a}$ \\ ${ }^{a}$ Warsaw University of Technology, Boboli 8, 02-525 Warsaw, Poland \\ ${ }^{b}$ Industrial Research Institute for Automation and Measurements PIAP, Jerozolimskie 202, 02-486 Warsaw, Poland
}

\begin{abstract}
Small values of external perturbation forces made it possible to use a torque magnetic drive as a control system. Today, satellite orientation systems use pulsed-type algorithm for discharge of an angular momentum accumulator. The feasibility of using other algorithms (continuous, continuous-pulsed and algorithm of torque magnetic drive operation using 'forecast' models of accumulated angular momentum and geomagnetic field) is not covered and not examined, although each has certain advantages for certain types of satellites and their conditions of operation in space. The article highlights satellites operating conditions in space, considers satellites orientation method using a torque magnetic drive and substantiates the necessity of satellite motion simulation with further establishing a technique for evaluation of orientation system given the effect of permanent magnetic and gravitational fields, the type of discharge of accumulated angular momentum, the height of trajectory and the nature of random perturbations.
\end{abstract}

DOI: 10.12693/APhysPolA.133.722

PACS/topics: 04.30.Tv, 07.05.Rm, 07.07.Df, 07.55.Ge

\section{Introduction}

In most cases, satellites in flight should be in a certain manner oriented in space. Whereas first satellites intended for scientific space research were uncontrolled, subsequent satellites had a range of devices on board providing their proper orientation in space.

Small values of external perturbation forces made it possible to use a torque magnetic drive (TMD) as a control system. Strict requirements for devices and elements used in satellites such as reliability, accuracy, light weight and power consumed shall be distinguished as properties of satellite control systems. Satellite orientation system consists of two parts: measuring and actuating. Further we will consider satellite orientation systems with an active actuator using magnetic control principle. The topical issue of today is to evaluate a certain algorithm for throwing off accumulated angular momentum (AAM) in the existing orientation systems, given the dynamics of satellite motion, the conditions of satellite operation in outer space and the availability of deterministic and random perturbations.

Today, satellite orientation systems use pulsed-type algorithm for discharge of an angular momentum accumulator. The feasibility of using other algorithms (continuous, continuous-pulsed and algorithm of TMD operation using 'forecast' models of AAM and geomagnetic field) is not covered and not examined, although each has certain advantages for certain types of satellites and their conditions of operation in space.

*corresponding author; e-mail: igor@mchtr.pw.edu.pl

\section{General information}

A basic orbital coordinate system has become the most widespread for satellites designed to study physical processes that occur on Earth. Axes of the system are directed as follows [1]: axis $O y_{0}$ - in local vertical direction (in the line that connects center of mass of the device and center of mass of the Earth); axis $O x_{0}$ - in transversal direction towards the satellite motion (in perpendicular direction towards the local vertical in orbital plane); axis $O z_{0}$ - in binormal vector direction in orbital plane so that the system $O x_{0} y_{0} z_{0}$ was right-handed. In general, mutual arrangement of axes of reference and fixed systems is determined by three Euler-Krylov angles $\gamma, \psi, \vartheta$, each of which defines a satellite orientation error. Figure 1 depicts Euler-Krylov angles relative to the axes of orbital system. Table I shows the values of direction cosines.

TABLE I

Direction cosines

\begin{tabular}{c|ccc}
\hline \hline Coordinate & $x_{0}$ & $y_{0}$ & $z_{0}$ \\
\hline$x$ & $\cos \vartheta \cos \psi$ & $\sin \vartheta \cos \psi$ & $-\sin \psi$ \\
$y$ & $-\cos \gamma \sin \vartheta+$ & $\cos \vartheta \cos \gamma+$ & $\sin \gamma \cos \psi$ \\
& $\sin \gamma \sin \psi \cos \vartheta$ & $\sin \vartheta \sin \psi \sin \gamma$ & \\
$z$ & $\sin \vartheta \sin \gamma+$ & $-\sin \gamma \cos \vartheta+$ & $\cos \psi \cos \gamma$ \\
& $\cos \vartheta \sin \psi \cos \gamma$ & $\cos \gamma \sin \vartheta \sin \psi$ &
\end{tabular}

If deviations are so small that $\sin (\gamma, \psi, \vartheta) \approx(\gamma, \psi, \vartheta)$ and $\cos (\gamma, \psi, \vartheta) \approx 1$ and their products are values of second order of infinitesimals, expressions for direction cosines are simplified and have the form shown in Table II:

In most cases, a torque device can be used in active satellite orientation systems. It represents a current coil placed on board of satellite, i.e a torque magnetic drive. 


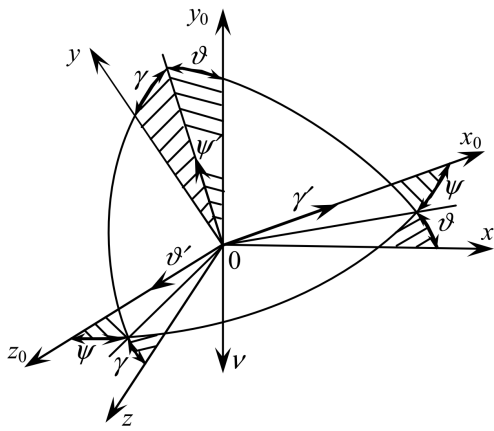

Fig. 1. Coordinate systems.

TABLE I

Approximate value of direction cosines

\begin{tabular}{c|ccc}
\hline \hline Coordinate & $x_{0}$ & $y_{0}$ & $z_{0}$ \\
\hline$x$ & 1 & $\theta$ & $\psi$ \\
$y$ & $-\vartheta$ & 1 & $\gamma$ \\
$z$ & $\psi$ & $-\gamma$ & 1
\end{tabular}

Figure 2 shows one of the possible schemes designed for satellite spatial orientation. It consists of three flat coils, three magnetometers for measuring EMF and a computing device $[2,3]$. Electric energy required for device operation comes from onboard power source.

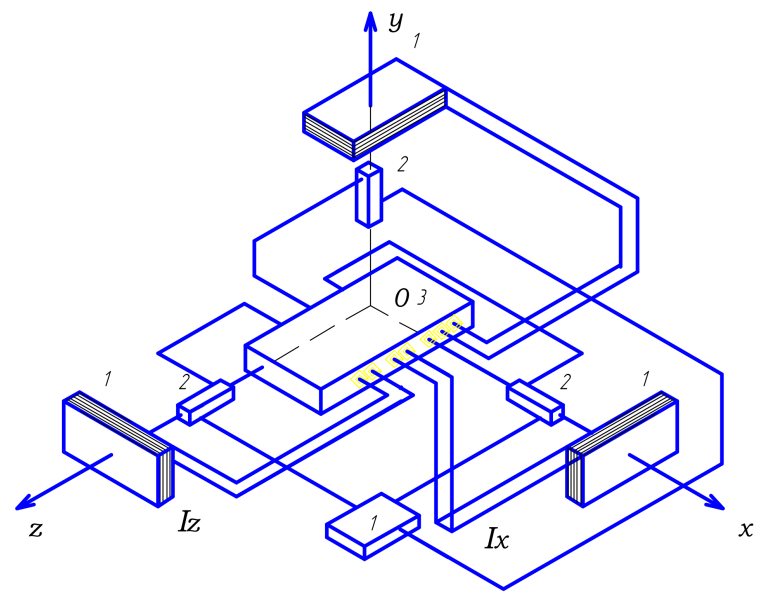

Fig. 2. Scheme of torque magnetic drive for satellite spatial orientation: 1 - a power coil; 2 - magnetometers; 3 - computing device; 4 - voltage source.

It should be noted that magnetic power coils of a satellite are rigidly connected to geometric axes of a satellite. Therefore, any (even minor) oscillations of a spacecraft result in electromotive force on measure windings of magnetic core.

This in turn leads to currents in power coils and moments that reset a spacecraft. That is, changing a single coordinate of control process affects the whole control process. Accordingly, each theoretical axis of a satellite has corresponding measuring and power coils. Let's consider a mathematical model of the process which uses a cross product of two magnetic fields, namely magnetic field of a satellite and magnetic field of a power coil.

A driving moment $z \boldsymbol{M}$ in such a device is created by interaction between magnetic moment of coil $\boldsymbol{P}$ and tension of local EMF $\boldsymbol{B}[1]: \boldsymbol{M}=\boldsymbol{P} \times \boldsymbol{B}$. Current flowing through the coil electromagnetic axis of which coincides with the axis $O_{x}$ is denoted by $I_{x}$, with the axis $O_{y}$ is denoted by $I_{y}$ and with axis $O_{z}$ is denoted by $I_{z}$. The magnetic moment of each coil would be:

$$
P_{x}=n S I_{x} ; \quad P_{y}=n S I_{y} ; \quad P_{z}=n S I_{z},
$$

where $n$ and $S$ are number of turns round and area of turns respectively.

Designing the expressions on the coordinate axes, we write corresponding expressions for currents to be passed through the coil to receive the desired values of a driving moment: $I_{x}=\frac{k}{n S}\left(B_{y} M_{z}-B_{z} M_{y}\right) ; I_{y}=\frac{k}{n S}\left(B_{z} M_{x}-\right.$ $\left.B_{x} M_{z}\right) ; I_{z}=\frac{k}{n S}\left(B_{x} M_{y}-B_{y} M_{x}\right)$, where $k=\frac{1}{B^{2}}$.

In general, TMD can serve as an independent actuator of a satellite orientation system, but in this case some specific features of a magnetic drive impose significant limitations on the system. Therefore, the most promising is considered to be the use of TMD in complex with actuator that includes not only magnetic drive but also a particular type of angular momentum (engines: flywheels, gyroscopes) [4-6]. In this case carrier of angular momentum serves as accumulator that average time of external perturbation moments on a satellite, which results in accumulation of angular momentum in the system "satellite - orientation system actuators" $\boldsymbol{H}=\int_{0}^{t} \boldsymbol{M}^{B}(t) \mathrm{d} \tau$, which shall periodically be reduced to a minimum. Discharge moment caused by a torque magnetic drive is used to compensate reactive perturbation moment occurring when throwing off angular momentum accumulated by the system. The following magnetic moment law is chosen at continuous discharge of angular momentum accumulators using magnetic drive [7]:

$$
\boldsymbol{p}=\frac{[k]}{B^{2} \boldsymbol{B} \times \boldsymbol{H}} .
$$

This algorithm makes it possible to use EMF for discharge of angular momentum accumulators, ensures minimization of energy costs, evenly charges onboard power sources. However, possibility of its use is limited, since the nature of magnetic field provides a constant process of continuous discharge on a limited class of satellite orbits. The [8] proposes a continuous-pulsed algorithm for discharge of angular momentum accumulators, allowing to use the positive features of algorithms described above and eliminate their weaknesses. Angular momentum carriers creating driving moments relative to two satellite axes, e.g. y and $z$, discharges continuously. Pulsed-type discharge algorithm is used for AAM discharge along the third axis, i.e. axis x. The (1) for magnetic moment of TMD in the continuous discharge regime is formed in accordance with the law: 


$$
\boldsymbol{p}=\frac{[k]}{B^{2} \boldsymbol{B} \times \boldsymbol{H}^{y z}},
$$

where $\boldsymbol{H}^{y z}$ is angular momentum accumulated by the system in the plane $y z$.

Proposes a new algorithm of TMD operation in satellite orientation system using 'predictive' models of AAM and geomagnetic field. The principle of the method is as follows. Components of the angular momentum accumulated along the satellite axes $H_{x}(t), H_{y}(t), H_{z}(t)$ bear information about the size and direction of perturbation moment $\boldsymbol{M}^{B}(t)$ affecting a satellite. Having a hypothetical model of perturbations affecting a satellite, we can obtain parameters of a 'forecast' model $H_{x}^{\mathrm{pr}}(t), H_{y}^{p r}(t)$, $H_{z}^{p r}(t)$, e.g. in the form of power series, based on processing of signals proportional to $H_{x}(t), H_{y}(t), H_{z}(t)$. Measurement of EMF component strength along the satellite axes $B_{x}(t), B_{y}(t), B_{z}(t)$ and processing of these signals on a certain time segment will enable to clarify the hypothetical model and obtain a 'forecast' model of the field $B_{x}^{p r}(t), B_{y}^{p r}(t), B_{z}^{p r}(t)$, e.g. in the form of Fourier series. On the basis of models $\bar{H}^{p r}(t), \bar{B}^{p r}(t)$ we can forecast operation conditions of a complex actuator on the future time segment, which, for example, equals to the period of satellite orbital revolution, and choose its optimum operation mode according to the energy cost minimum criterion. Application of this method will reduce energy consumption and mass of angular momentum accumulator.

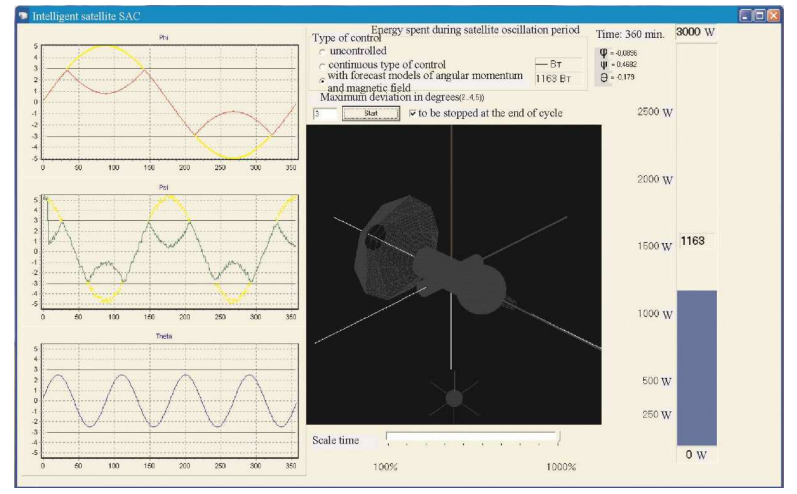

Fig. 3. Simulation of a satellite orientation method with forecast models of magnetic field and accumulated angular momentum.
Figure 3 shows a computer simulation of a satellite control method with forecast models (limitations) of the moment along all three axes. To evaluate effectiveness of a control method, choose the program mode "With forecast models of kinetic moment and magnetic field", put a tick in the block "Stop at the end of the cycle" and press "Start" (Fig. 3). Red, green, and blue lines on the graphs show real angles of deviation, and yellow line shows deviations that would have been if there was no control.

As can be seen, this method uses less energy, thus increasing the period of controlled stay of satellite in orbit.

\section{Conclusion}

This control method does not allow a satellite to deviate by more than $2.9^{\circ}$. Having carried out several simulations, it turned out that power consumption arithmetic mean is $1200 \mathrm{~W}$. At the simulation of $\pm 3^{\circ}$ satellite deviation limit, as can be seen on angles deviation graphs, roll angle and yaw angle are within $\pm 5^{\circ}$, and pitch angle is within $\pm 2.5^{\circ}$. At the allowed limit of $\pm 3^{\circ}$ the pitch angle is not regulated, which saves power.

\section{References}

[1] Don Lancaster, Electronics Now 67 9, 65 (1996).

[2] L. Richard, in: Int. Conf. Appl. Electr., Pilsen 2006, p. 113.

[3] A. Praczukowska, M. Nowicki, I. Korobiichuk, R. Szewczyk, J. Salach, Eastern-European J. Enterprise Techn. 6/5 (78), 4 (2015).

[4] I. Korobiichuk, O. Bezvesilna, M. Kachniarz, A. Tkachuk, T. Chilchenko, Adv. Intell. Syst. Comp. 543, 481 (2017).

[5] I. Korobiichuk, V. Karachun, V. Mel'nick, M. Kachniarz, Metrol. Meas. Syst. 24, 357 (2017).

[6] I. Korobiichuk, M. Nowicki, R. Szewczyk, JAMRIS, 9, 47 (2015).

[7] C. Moreland, Poptronics 2, 29 (2001).

[8] A. Zakharova, D. Shostachuk, Conf. Current Trends Young Sci. Res. Zhytomyr 2016, p. 131. 